\title{
Opistborchis lomeensis n. sp. Douve biliaire de l'Amphibien Aubria subsigillata au Togo
}

\author{
par R. BOURGAT * et Cl. COMBES ** \\ (Collaboration technique: Noulagnon Bossou) \\ * Université du Bénin, B.P. $n^{\circ} 1515$, Lome, Togo \\ ** Centre Universitaire, F 66025 Perpignan
}

\section{Résumé.}

Opisthorchis lomeensis n. sp. (Trematoda, Opisthorchiidae) est décrit de la vésicule biliaire d'Aubria subsigillata (Anura, Ranidae). Il se caractérise principalement par son tégument non spinulé, son rapport ventousaire inférieur à 1, un pré-pharynx court, un ovaire nettement séparé des testicules et des follicules vitellogènes débutant un peu en arrière de l'acetabulum pour se terminer au niveau du bord antérieur de l'ovaire. $O$. lomeensis est le premier Opisthorchiidae adulte connu d'Amphibien.

\section{Summary.}

Opisthorchis lomeensis n. sp., a new fluke of the gall bladder of the amphibian Aubria subsigillata in Togo.

Opisthorchis lomeensis n. sp. (Trematoda, Opisthorchiidae) is described from the gall bladder of Aubria subsigillata (Anura, Ranidae). It is mainly characterized by the smooth tegument, the sucker ratio less than one, the short prepharynx, the ovary clearly separated from the testis, and the vitelline follicles extending from a level situated a little behind the acetabulum to the level of the anterior edge of the ovary. O. lomeensis is the first adult Opisthorchiidae to be described from an Amphibian. 
Aubria subsigillata (Duméril) est un Amphibien Anoure Ranidae typiquement inféodé aux biotopes forestiers d'Afrique. Nous en avons capturé 19 exemplaires dans une forêt galerie du Togo ; 5 d'entre eux hébergeaient respectivement $1,6,8,18$, 20 Douves appartenant à une espèce nouvelle pour la Science, dont nous donnons la description ci-après.

\section{Description}

Hôte: Aubria subsigillata (Duméril).

Localité: Kovié, $30 \mathrm{~km}$ au nord de Lomé (Togo).

HABITAT : vésicule et canaux biliaires.

MATÉRIEL DE DESCRIPTION : 30 exemplaires montés in toto (mensurations de 4 d'entre eux dans le tableau $I$ ).

Tableau I. - Dimensions (en $\mu$ ) de 4 exemplaires adultes de Opistorchis lomeensis n. sp.

\begin{tabular}{|c|c|c|c|c|}
\hline Longueur $\quad \ldots \ldots \ldots \ldots \ldots \ldots \ldots$ & 4200 & 4440 & 2600 & 4900 \\
\hline Largeur maximale $\ldots \ldots \ldots \ldots \ldots$ & 390 & 750 & 420 & 450 \\
\hline Largeur niveau V.V. ............ & 225 & 340 & 210 & 190 \\
\hline v.o. $\quad \cdots \cdots \cdots \cdots \cdots \cdots$ & $132 \times 135$ & $135 \times 135$ & $102 \times 93$ & $130 \times 135$ \\
\hline$\ldots \ldots \ldots \ldots$ & $150 \times 125$ & $168 \times 180$ & $105 \times 99$ & $140 \times 140$ \\
\hline Distance V.O.-V.V. .............. & 620 & 600 & 460 & 390 \\
\hline Rapport V.O./V.V. $\ldots \ldots \ldots \ldots \ldots$ & 0,97 & 0,77 & 0,95 & 0,95 \\
\hline Longueur prépharynx $\ldots \ldots \ldots \ldots$ & 3 & 0 & 5 & 3 \\
\hline Pharynx $\ldots \ldots \ldots \ldots \ldots$ & $70 \times 72$ & $66 \times 79$ & $52 \times 45$ & $70 \times 72$ \\
\hline Esophage $\ldots \ldots \ldots \ldots \ldots \ldots$ & $155 \times 22$ & $225 \times 33$ & $102 \times 21$ & $120 \times 30$ \\
\hline Diamètre Cæcums $\ldots \ldots \ldots \ldots \ldots$ & $20-75$ & $35-90$ & $25-75$ & $20-75$ \\
\hline Distance Caec-Extr. post. $\ldots \ldots \ldots$ & 190 et 275 & 135 et 190 & 115 et 135 & 150 et 210 \\
\hline Ovaire $\quad \ldots \ldots \ldots \ldots \ldots \ldots \ldots$ & $132 \times 165$ & $105 \times 180$ & $105 \times 138$ & $180 \times 210$ \\
\hline Réceptacle séminal $\ldots \ldots \ldots \ldots \ldots$ & $100 \times 180$ & $150 \times 240$ & $143 \times 121$ & $130 \times 195$ \\
\hline Testicule antérieur $\ldots \ldots \ldots \ldots \ldots$ & $210 \times 175$ & $150 \times 250$ & $150 \times 165$ & $285 \times 305$ \\
\hline Testicule postérieur...... & $190 \times 210$ & $160 \times 200$ & $168 \times 168$ & $300 \times 300$ \\
\hline Distance ov.-test. ant. $\ldots \ldots \ldots \ldots$. & 195 & 300 & 120 & 480 \\
\hline Distance test. post.-extr. post. $\ldots$. & 450 & 450 & 255 & 475 \\
\hline 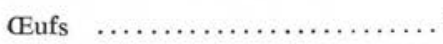 & $21-24 \times 11-13$ & $21-24 \times 11-13$ & $21-24 \times 11-13$ & $21-24 \times 11-13$ \\
\hline
\end{tabular}

CORPS : aplati, très allongé, effilé antérieurement.

TÉGUMENT : mince, glabre. 
Ventouses: ventouse orale subterminale ventrale; acetabulum très proche de l'extrémité antérieure de l'animal.

APPAREIL DIGESTIF : prépharynx réduit ou nul, pharynx bien développé. Esophage fin et long. Bifurcations caecales à mi-distance entre la ventouse orale et l'acetabulum. Caecums de calibre un peu irrégulier se terminant au voisinage immédiat de l'extrémité du Ver.

APPAREIL gÉNITAL MALE: testicules ronds ou légèrement ovoïdes, disposés en oblique, au $1 / 5^{\circ}$ postérieur du corps environ. Canaux déférents se réunissant en avant de l'ovaire pour former le spermiducte qui se différencie en vésicule séminale contournée. Pas de poche du cirre. Pore génital médioventral, immédiatement pré-acétabulaire.

APPAREIL GÉNITAL FEMElle: ovaire médian. subsphérique, situé au $1 / 4$ postérieur du corps. Distance ovaire-testicule antérieur relativement importante. Réceptacle séminal volumineux immédiatement post-ovarien. Canal de Laurer présent. Utérus débutant en arrière de l'ovaire puis formant des boucles stratifiées peu irrégulières dans la totalité du champ intercaecal entre l'ovaire et l'acétabulum, et aboutissant au pore génital commun. Vitellogènes en petits follicules réguliers formant une mince bande dans les champs extracaecaux depuis un niveau situé un peu en arrière de l'acétabulum jusqu'au niveau du bord antérieur de l'ovaire. Eufs jaune paille, très nombreux, à clapet.

APPAREIL EXCRÉTEUR: vésicule excrétrice sigmoïde passant entre les deux testicules et se bifurquant en $\mathrm{T}$ dissymétrique en avant du testicule antérieur. Elle reçoit 2 canaux collecteurs très visibles sur le vivant qui cheminent dans les champs extra-caecaux jusqu'au niveau du pharynx.

\section{Discussion}

Par l'ensemble de ses caractères, le Digène que nous décrivons est un Opisthorchiidae et prend place dans le genre Opisthorchis Blanchard, 1895. Ce genre compte actuellement (Yamaguti, 1971) 6 espèces parasites de Poissons, dont 1 en Afrique (Egypte), 20 espèces parasites d'Oiseaux, dont 3 signalées en Afrique ( 2 en Egypte, 1 en Rhodésie), 6 espèces parasites de Mammifères dont 1 en Afrique (Somalie). Le

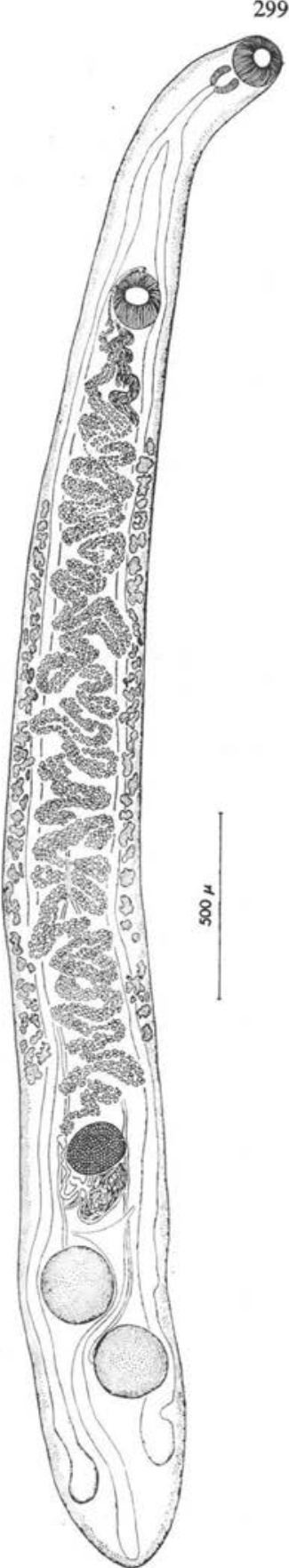

FIG. 1. - Opisthorchis lomeensis n. sp.: vue ventrale. 
genre Opisthorchis n'est donc d'aucun Amphibien dans le monde, ni d'aucun Vertébré d'Afrique Occidentale.

Sur le plan morphologique et anatomique nos spécimens ne s'identifient à aucune espèce connue; d'autre part, il est certain, vu sa fréquence et son état de maturité parfaite que notre Digène n'est ni un parasite accidentel ni un parasite transfuge et que son hôte définitif normal est réellement un Amphibien. Dans ces conditions, nous proposons la création de l'espèce Opisthorchis lomeensis n. $s p$.

La diagnose de l'espèce peut se résumer ainsi; tégument non spinulé; rapport v.o./v.v. inférieur à 1 ; prépharynx très court ; bifurcation caecale nettement préacetabulaire; espace entre ovaire et testicule antérieur important; testicules non lobés, vitellogènes n'atteignant pas l'acetabulum vers l'avant et se terminant au niveau de l'ovaire en arrière.

\section{Conclusion}

Les Opisthorchiidae étant jusqu'ici connus à l'état adulte seulement de Poissons, Reptiles, Oiseaux et Mammifères, $O$. lomeensis est le premier représentant de cette famille chez un Amphibien. Nous soulignerons en outre que les Trématodes hépatobiliaires d'Amphibiens actuellement décrits sont au nombre de quatre seulement:

\section{Plagiorchiidae:}

Glypthelmins intermedia (Caballero, Bravo-Hollis, Zerecero, 1944) chez Bufo marinus en Amérique Centrale.

Glypthelmins vesicalis (Ruiz et Leao, 1942) chez Bufo marinus au Brésil.

Xenopodistomum xenopodis Macnae, Rock et Makowski, 1973 chez Xenopus laevis en Afrique du Sud.

\section{Lecithodendriidae:}

Pleurogenes hepaticola Grabda-Kazubska, 1972 chez Rana esculenta, Rana ridibunda et Rana arvalis en Pologne.

En ce qui concerne le cycle biologique, et au regard des connaissances actuelles sur le cycle des Opisthorchiidae, on peut penser que les métacercaires se forment soit chez les Poissons, soit chez des Tétards d'Amphibiens. Dans ce deuxième cas, qui nous paraît le plus vraisemblable, les Aubria subsigillata adultes s'infesteraient en consommant leurs propres jeunes ou ceux d'Anoures sympatriques.

\section{Bibliographie}

Caballero y C. (E.), Bravo-Hollis (M.) et Zerecero (M. C.), 1944. - Estudios helmintologicos de la region oncocercosa de Mexico y de la República de Guatemala. I. Ann. Inst. Biol., 15, 59-72. 
RuIZ (J. M.) et LeAo (A. T.) 1943. - Notas helmintologicas. IV. Choledocystus vesicalis n. sp. parasita du vesicula biliar de Bufo marinus (L.). Mém. Inst. Butantan, 16, 209-217.

Grabda-Kazubska (B.), 1972. - Pleurogenes hepaticola sp. n. (Trematoda, Pleurogenidae), a parasite of the biliary duct of Rana esculenta L. in Poland. Acta parasitol. polonica, 20, 45-54.

Macnae (W.), Rock (L.), et Makowski (M.), 1973. - Platyhelminths from the South African Clawed Toad, or Platanna (Xenopus laevis). J. Helminthology, 47, 199235.

Yamaguti (S.), 1971. - Synopsis of the Digenetic Trematodes of Vertebrates Vol. I, Keigaku Publishing Co., Tokyo, 1074 p. 